\title{
Research on Emotional Design of Furniture in Space from the Perspective of Interaction
}

\author{
Zhou Jiahui \\ Changchun University of Technology, Jilin, China
}

Keywords: interaction; furniture; emotional design

Abstract: Furniture can express the emotion of furniture itself while expressing human emotion. Therefore, the introduction of emotional design concept into furniture has become an urgent need for social development, which is of great significance. The concept of interaction design has been applied more and more widely, among which the application of interaction technology in the home environment has been on the rise. This paper briefly expounds the necessity of emotionalized furniture design, mainly introduces the concept of emotionalized furniture design and studies the emotionalized furniture design method from the perspective of interaction, so as to enhance people's emotional and spiritual communication and realize the optimization of furniture design.

\section{Introduction}

Furniture is a necessity in people's daily life, and its design directly affects people's emotions. Modern furniture design is more and more emphasis on technical and material, which largely ignores the emotionality of furniture, and there are problems such as patterning, too modern and simple. Therefore, the modern furniture design should consider the interaction between the spirit and emotion of furniture and people, and more reflect people's emotional expression, making it a kind of "sensible product", which will be the trend of furniture design.

\section{The necessity of emotional design of furniture}

It can be seen from the history of the changes of ancient Chinese furniture that people's behaviors and lifestyles play a decisive role in the development direction of furniture design. In today's society where the pace of life is accelerating, people's lifestyles have undergone earth-shaking changes. How to meet the high requirements of people's quality of life through furniture design, and promote the interaction between people and furniture and good communication between people has become a new goal of contemporary interior furniture design. Under such requirements, the emergence of emotional design in interior furniture has become inevitable ${ }^{[1]}$. 


\section{Overview of furniture emotional design}

\subsection{Definition of furniture emotional design}

The emotional design of furniture is based on the function and use, from the perspective of human physiology and psychology, injecting a multi-pronged "sensing factor" into the furniture design, paying attention to the symbolic, artistic, cultural and individual characteristics of furniture. According to the differences in people's consumption psychology, using visual symbols such as design language and aesthetic rules, the emotions are transformed into recognizable furniture carriers, so that people can find the destinations that satisfy their emotional needs, thus obtaining physiological comfort and psychological. The joy of sympathy and rationality in the emotional interaction between man and furniture.

\subsection{Three levels of expression of furniture emotional design}

In the book "Emotional Design", Donald Norman analyzes the three levels of human cognitive items from the perspective of cognitive psychology: instinct level, behavior level and reflection level.

First, the instinct level. The emotional design of furniture instinct mainly refers to the emotional design of furniture modeling. It integrates the shape, color perception and material texture of furniture through certain design methods to create products with practical functions and aesthetic value. The subject produces an emotional experience in the process of using the product. In the emotional design of indoor furniture, different morphological elements are selected for different use subjects to meet the different needs of each group. Appearance can promote emotional interaction between people and products, people, and stimulate consumers' desire to buy. The figurative form of furniture is the imitation and deformation of nature, and the emotional association is given by imitating objects. In the product design, you can convey the emotion of the product through different color combinations, and use the reasonable color to give people a fresh feeling and add a sense of life. The flower seat is one of the most eye-catching designs in the Milan Design Exhibition, from the Japanese designer Tokujin Yoshika. When someone sits down, the flower buds will open automatically, forming a flower cluster with graceful blooms. People will be tightly surrounded by flower clusters. When the person leaves the seat, the flower cluster will revert to the previous flower creation type, delicate details. impressive. Yoshika hopes that this seat will give people a sense of well-being like receiving flowers ${ }^{[2]}$. Table 1 shows the views of some people on the flower seat.

Table 1 Flower seat design evaluation results

\begin{tabular}{ccc}
\hline Evaluation index & Grade & Number of people \\
\hline \multirow{2}{*}{ Form } & excellent & 83 \\
& good & 8 \\
\multirow{3}{*}{ Colour } & medium & 9 \\
& excellent & 75 \\
\multirow{3}{*}{ Material } & good & 20 \\
& medium & 5 \\
& excellent & 80 \\
Psychological feeling & good & 5 \\
& medium & 15 \\
& great & 90 \\
& good & 6 \\
& general & 4 \\
\hline
\end{tabular}


Second, the behavioral level. The establishment of emotion between people and products is based on the functional utility of the product. The emotions in the use of the subject reflect the function of the product. Products with good use functions will be handy when used, resulting in positive and positive psychological feelings. Therefore, products that embody rich inner feelings are easier to use. This is why the ease of use of the function is always associated with the emotion in the design.

Third, the level of reflection. Through the interaction with people, furniture conveys information and expresses its ability as an artifact to convey feelings and become an important medium for communication and interaction between people and people. The interior furniture design is not only to achieve the function of the furniture, but also to achieve the sense of dependence and pleasure between the subject and the object. Therefore, the emotional integration allows the furniture design to have more spiritual and emotional value to meet the increasing needs of people. As shown in Figure 1, the pregnant chair and the large chair "grow" a small chair. The two chairs are independent and interdependent, and they are touching and moving ${ }^{[3]}$.

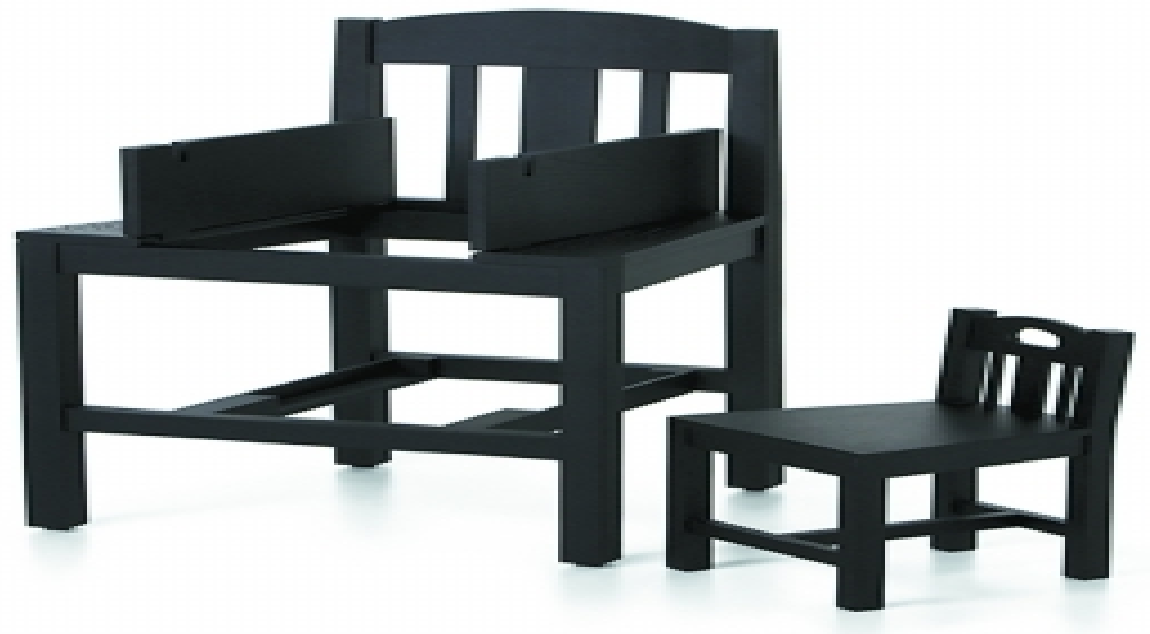

Figure 1 Pregnant chair

\subsection{Emotional design of furniture space environment}

The shape, function and emotional experience of furniture in emotional design are relatively intuitive and important factors for people. Another aspect of the importance of interior furniture design is that the emotional design of the space environment is often overlooked. It is very common to study furniture as an individual, but in fact furniture is not only a display of indoor space, but also an important part of the indoor space environment. Therefore, research furniture must study the corresponding space environment.

In the interior furniture design, the indoor environment is one of the factors that affect people's behavior and emotions. The shape and function of the furniture affect the style and function of the overall environment, and form an emotional exchange and interaction with the space environment. Therefore, it is necessary to make the product become the link between the space environment and the human through the emotional design of the furniture, so that the furniture form and the scale relationship form an emotional transition between the space environment and the use subject. 


\section{Emotional design method of furniture from the perspective of interaction}

\subsection{Guided by multiple theories}

Modern furniture product design is completed under the guidance of a variety of design theories. In order to achieve the usability of products and the unique user experience goals of furniture products, it is necessary to combine general design, interaction design, experience design and other theories commonly used in furniture product design under the background of emotional design concept.

\subsection{Set a moderate experience with the user as the center}

Incorporating interactive design concepts into furniture product design is a design approach and trend, but not all furniture products need to be interactive products. Norman mentioned in Design Psychology that good design does not necessarily have to have many powerful functions, nor is it equivalent to the accumulation of technology. Therefore, to avoid the design of unnecessary interactive furniture products, you need to do something first-stop the designer's subjective temperament, start from the user, observe the user's behavior, and determine the user's actual needs. After identifying the target user base, design with them and change the design at any time ${ }^{[4]}$.

\subsection{Adopting tangible technology in the context of the environment}

Furniture products are often subject to environmental constraints, so interactive furniture products should be designed with attention to the impact of the furniture environment on the product. Starting from the basic characteristics of furniture products, that is, safe, comfortable and applicable, techniques that are excessively exaggerated and not suitable for the environment should be avoided. Not all high-tech products are suitable for the design of furniture products. Some products that use solar energy or environmental protection as the theme can not only achieve the expected results in practical applications, but indirectly cause waste of resources. Therefore, under the premise of fully considering the use of the environment, the product should be designed in the user's use process ${ }^{[5]}$.

\subsection{Return the initiative of the product to the user}

When a product is designed, it is usually designed as a complete product, and the user's product is rarely changed. When the furniture product is designed, the designer can try to "half" the product design and return the "other half" to the user, who decides the style and function of the product. "Half" does not mean incomplete, but it invites users to complete the remaining "half" in an incomplete form. When the user designs the rest, he may feel the experience that the product itself cannot bring. This kind of interaction makes the user not face a product that he has no autonomy, but participates in making the product available for himself.

\section{Conclusions}

The emotional design of furniture is a specific application of design psychology in furniture design. People-oriented design, emotional-oriented experience design has become a trend of attention. Modern furniture should be a combination of science and art, technology and humanity. Science and technology give modern furniture a solid structure and good function; While the emotional factor of art and humanity is that furniture is full of beauty, full of fun and vitality, and it 
becomes a bond of harmony between people and design. People's likes and dislikes of furniture depend on many factors. In modern fast-paced life, people are particularly sensitive to design language such as emotion and care. This is the characteristics of the times rich in furniture, furniture design should pay more attention to the subjective needs that emotional needs, and then design a furniture product that can say people's "heart".

\section{References}

[1] Mao Wei, Zhu Lishan. Emotional Design of Interior Furniture Based on Interactive Concept[J]. Art Education, 2016(6): 204-205.

[2] Lu Rongxue. Research on the emotional design of furniture [D]. Shenyang Aerospace University, 2016.

[3] Li Jing, Huang Sheng. Research on Emotional Design of Modern Bamboo Furniture[J]. Furniture \& Interior, 2015(1).

[4] Wang Lina, Li Binbin. Research on Emotional Design of User Experience under the View of Interactive Wall[J]. Journal of Public Art, 2011(22): 46-46.

[5] Jiang Yue. Research on Emotional Design of Smart Home[J]. Furniture \& Interior, 2018(2). 